\title{
EXCEL® COMO FERRAMENTA DIDÁTICA DE APOIO NA ANÁLISE DE PROCESSOS DE EXTRAÇÃO CONTÍNUA SÓLIDO- LÍQUIDO EM CONTRACORRENTE
}

\author{
C. A. S. FERREIRA ${ }^{1}$, O. C. MOTTA LIMA ${ }^{1}$ \\ ${ }^{1}$ Universidade Estadual de Maringá, Departamento de Engenharia Química \\ E-mail para contato: casf.eq@gmail.com
}

\begin{abstract}
RESUMO - A extração contínua é um processo industrial bastante versátil. A determinação do número de estágios de um processo contracorrente pode ser feita atra vés do procedimento gráfico e do método analítico. Esses métodos necessitam de dados termodinâmicos ou de gráficos de equilíbrio e consomem um tempo considerável para operações com um número alto de estágios. Este trabalho segue a ideia do projeto de modernização das disciplinas de operações unitárias e propõe o uso do EXCEL e da interface do Visual Basic na determinação do número de estágios, além de oferecer uma plataforma de aprendizado simples para os métodos apresentados. Os resultados são apresentados nas formas de gráficos e tabelas, facilitando o entendimento. Dessa forma, pode-se conciliar a teoria e a prática em sala de aula.
\end{abstract}

\section{INTRODUÇÃO}

A extração sólido-líquido é uma operação unitária bastante utilizada em indústrias químicas. Como exemplo, podemos citar a extração do café solúvel e a extração de óleos vegetais. Este processo consiste na separação de um ou mais constituítes solúveis presentes em uma mistura de sólidos através da ação de um solvente líquido (PERRY e CHILTON, 1980; FOUST et al., 1982).

A fim de aumentar a quantidade de soluto extraído e a produção de uma solução rica neste, utiliza-se uma bateria de extratores em série. A extração contínua em contracorrente é então utilizada com a finalidade de se economizar solvente e na obtenção de soluções mais concentradas do constituinte desejado (MOTTA LIMA e PEREIRA, 2010).

Ao trabalhar com um processo de extração que exija um número muito alto de estágios, os cálculos e procedimentos para determinação deste número acabam tornando-se muito dispendiosos. Neste contexto, este trabalho tem como objetivos a implementação de uma ferramenta de apoio na realização destes cálculos e procedimentos, bem como a facilitação do ensino da metodologia.

Sendo assim, apresenta-se uma proposta de utilização do software EXCEL® na análise de processos de extração contínua em contracorrente através do procedimento gráfico. A utilização desta ferramenta, permitiria ao professor a exploração de um maior número de situações em sala de aula.

Além disso, esta ferramenta apresenta um tutorial detalhado de uso, que consiste numa 
apresentação do procedimento gráfico utilizado e, portanto, pode ser utilizada pelos alunos em seus computadores pessoais, seja como ferramenta de apoio à solução de problemas selecionados, seja como ferramenta de revisão da metodologia.

\section{EXTRAÇÃO CONTÍNUA EM CONTRACORRENTE}

Numa bateria de extração funcionando em contracorrente, as duas fases entram por extremidades opostas da bateria e, em cada estágio, circulam em sentidos contrários. Os estágios são numerados no sentido de movimento do fluxo mais pesado.

O material a extrair é considerado proveniente de um tanque de armazenagem e o fluxo deste material é representado como se procedesse de um estágio de ordem zero, daí ser representado por $\mathrm{L}_{0}$, enquanto que o solvente é considerado como proveniente de um tanque situado a lém do estágio de ordem $\mathrm{N}$, daí o fluxo do solvente ser representado pela notação $\mathrm{V}_{\mathrm{N}+1}$.

A figura 1 apresenta um esquema típico de uma série de extratores contínuos em contracorrente (MOTTA LIMA e PEREIRA, 2010).

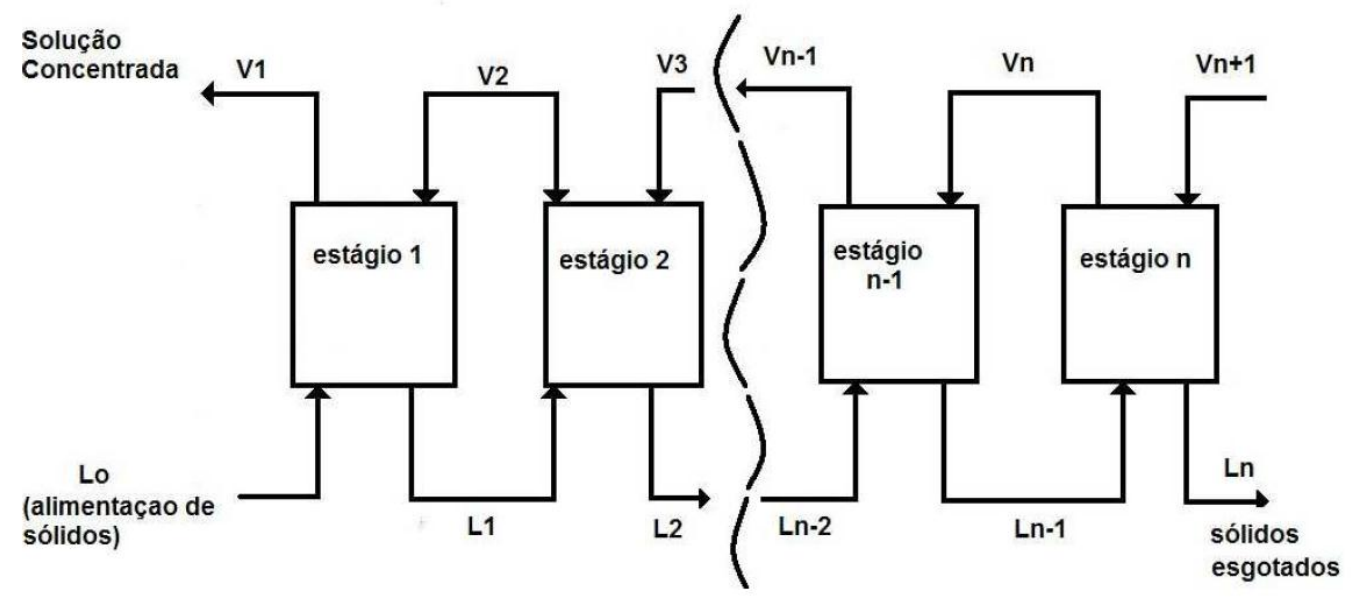

Figura 1 - Esquema de uma bateria de extratores contínuos em contracorrente.

Um balanço material total do processo como um todo fornece

$$
L_{0}+V_{N+1}=L_{N}+V_{1}
$$

enquanto que um balanço material parcial do soluto fornece

$$
x_{A, 0} \cdot L_{0}+y_{A, N+1} \cdot V_{N+1}=x_{A, N} \cdot L_{N}+y_{A, 1} \cdot V_{1},
$$

e um balanço material parcial do solvente fornece

$$
x_{S, 0} \cdot L_{0}+y_{S, N+1} \cdot V_{N+1}=x_{S, N} \cdot L_{N}+y_{S, 1} \cdot V_{1} \text {. }
$$




\section{9 a 22 de outubro de 2014 \\ Florianópolis/SC}

As equações (1), (2) e (3) são então utilizadas na resolução dos problemas relacionados.

Determinação do número de estágios: A determinação do número teórico de estágios pode ser realizada pelo método analítico, mediante balanços materiais em cada estágio e através dos dados experimentais de retenção de solução pela mistura sólida. Porém, este método torna-se demorado e cansativo para uma longa bateria de extratores. Métodos gráficos não somente simplificam os cálculos, como também diminuem os erros e assinalam de um modo mais claro as variações que possam ocorrer e seus efeitos sobre a operação.

Cálculo em diagramas retangulares: Neste método, o diagrama tem como abscissas $X=A /(A+S)$, ou seja, a relação existente entre a massa de soluto e a massa de solução, sendo que o valor de $\mathrm{X}$ engloba todo o soluto associado com uma mistura, incluindo não só a parcela dissolvida na solução retida pelos sólidos, como também a parcela do soluto não dissolvido ou adsorvido. Para as ordenadas, $\mathrm{Y}=\mathrm{B} /(\mathrm{A}+\mathrm{S})$, ou seja, a relação entre a massa de sólidos inertes e a massa de solução.

Uma vez que, atingido o equilíbrio na extração sólido-líquido, a solução ou mistura sobrenadante e a pasta formada tem a mesma concentração do soluto, X. As linhas que unem a composição da solução sobrenadante com a composição da pasta num diagrama retangular serão retas verticais paralelas ao eixo Y. Estas linhas recebem o nome de linhas de amarração.

Da equação (1), obtemos

$$
L_{0}-V_{1}=L_{N}-V_{N+1} \text {. }
$$

A diferença entre os fluxos que se cruzam, representada pela equação (4), é constante e denominada fluxo líquido. Designa-se o símbolo $\Delta$ para esta diferença. Portanto, tem-se que

$$
\Delta=L_{0}-V_{1}=L_{N}-V_{N+1}
$$

O fluxo líquido $\Delta$, por ser uma diferença entre dois fluxos, é o que chamamos, graficamente, de ponto de diferença. Como $\Delta$ é uma quantidade matemática que não existe fisicamente, suas coordenadas podem ter valores positivos ou negativos, dependendo se $\mathrm{L}_{0}>\mathrm{V}_{1}$ ou $\mathrm{L}_{0}<\mathrm{V}_{1}$.

A equação (5) mostra que, graficamente, o ponto $\Delta$ e os pontos $\mathrm{L}_{0}$ e $\mathrm{V}_{1}$ deverão estar sob uma mesma reta. Analogamente, $\Delta, \mathrm{L}_{\mathrm{N}}$ e $\mathrm{V}_{\mathrm{N}+1}$ deverão estar sob outra mesma reta. Dessa forma, $\Delta$ pode ser encontrado através da intersecção das retas formadas por $L_{0}$ e $V_{1}$ e por $L_{N}$ e $V_{N+1}$.

Como o fluxo líquido $\Delta$ é constante para todo estágio, utilizamos este ponto, em conjunto com as retas de amarração, para determinar o número teórico de estágios. Este procedimento é realizado da seguinte maneira: (a) plota-se $\mathrm{L}_{0}, \mathrm{~V}_{1}, \mathrm{~L}_{\mathrm{N}}$ e $\mathrm{V}_{\mathrm{N}+1}$; (b) prolonga-se as retas $\mathrm{L}_{0} \mathrm{~V}_{1}$ e $\mathrm{L}_{\mathrm{N}} \mathrm{V}_{\mathrm{N}+1}$ até cruzaremse no ponto $\Delta$; (c) a partir de $\mathrm{V}_{1}$, sobe-se verticalmente até a curva de retenção e determina-se $\mathrm{L}_{1} ;(\mathrm{d})$ a partir de $\mathrm{L}_{1}$, traça-se uma reta até o ponto $\Delta$; (e) do cruzamento da reta traçada em (d) e o eixo das abscissas, obtém-se $\mathrm{V}_{2}$; (f) repete-se (c), (d) e (e) até que uma das retas verticais (linhas de amarração) cruze a reta $\mathrm{L}_{\mathrm{N}} \mathrm{V}_{\mathrm{N}+1}$. A figura 2 apresenta este procedimento. 


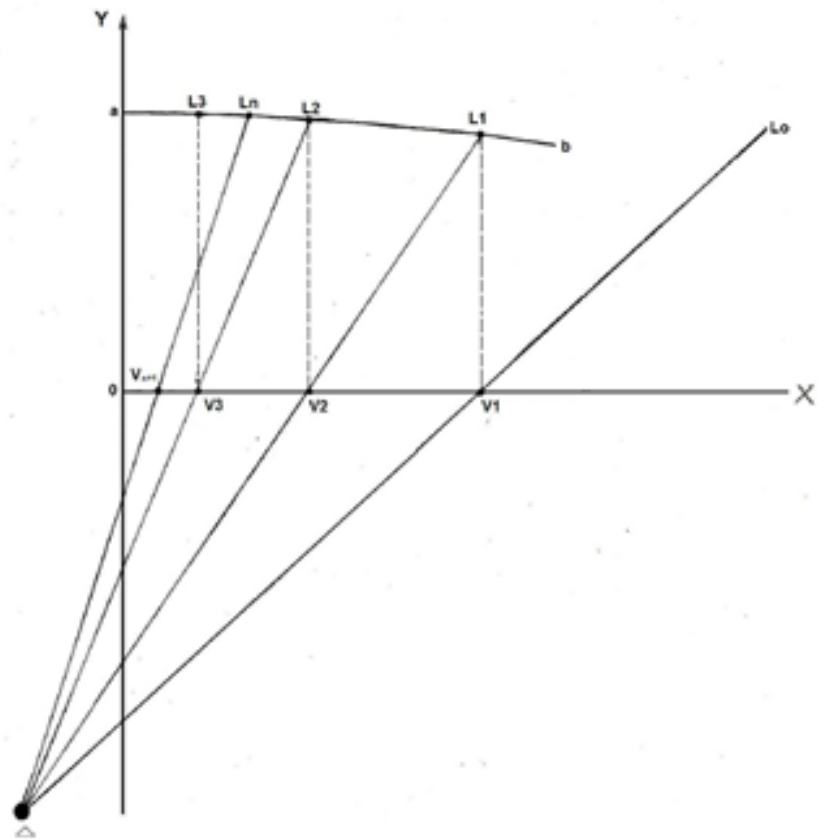

Figura 2 - Determinação gráfica do número teórico de estágios em diagrama retangular.

\section{EXCEL® NA ANÁLISE E RESOLUÇÃO DE PROBLEMAS DE ESL}

Ajuste dos parâmetros do modelo: Para realizar o cálculo em um diagrama retangular, primeiramente ajusta-se um modelo matemático ao conjunto de dados experimentais, a fim de se dispor de uma curva contínua. Sendo assim, de posse dos dados experimentais escrevemos $\mathrm{X}=\mathrm{A} /(\mathrm{A}+\mathrm{S})$ e $\mathrm{Y}=\mathrm{B} /(\mathrm{A}+\mathrm{S})$. Escrevemo s então uma nova variável, função polinomial de $\mathrm{X}$, conforme a equação (6).

$$
Y_{k}^{*}=A_{0}+A_{1} \cdot X_{k}+A_{2} \cdot\left(X_{K}\right)^{2}+A_{3} \cdot\left(X_{K}\right)^{3},
$$

onde $X_{k}$ representa a abscissa do ponto experimental $\mathrm{k}$ e $A_{0}, A_{1}, A_{2}$ e $A_{3}$ são as constantes do polinômio. Escrevemos então a seguinte função-objetivo

$$
F_{o b j}=\sum_{k=1}^{k=n}\left(Y_{k}-Y_{k}^{*}\right)^{2},
$$

onde $\mathrm{n}$ representa o número total de pontos experimentais.

Com o uso do SOLVER do EXCEL $\AA$, variam-se as constantes $A_{0}, A_{1}, A_{2}$ e $A_{3}$ a fim de minimizar a função objetivo dada pela equação (7) e, deste modo, encontra-se o polinômio que melhor se ajusta ao conjunto de dados.

Algoritmo para o cálculo em diagrama retangular: A forma geral do algoritmo utilizado no cálculo do número de estágios pode ser resumida pelo pseudocódigo pode ser visto na figura 3 . 


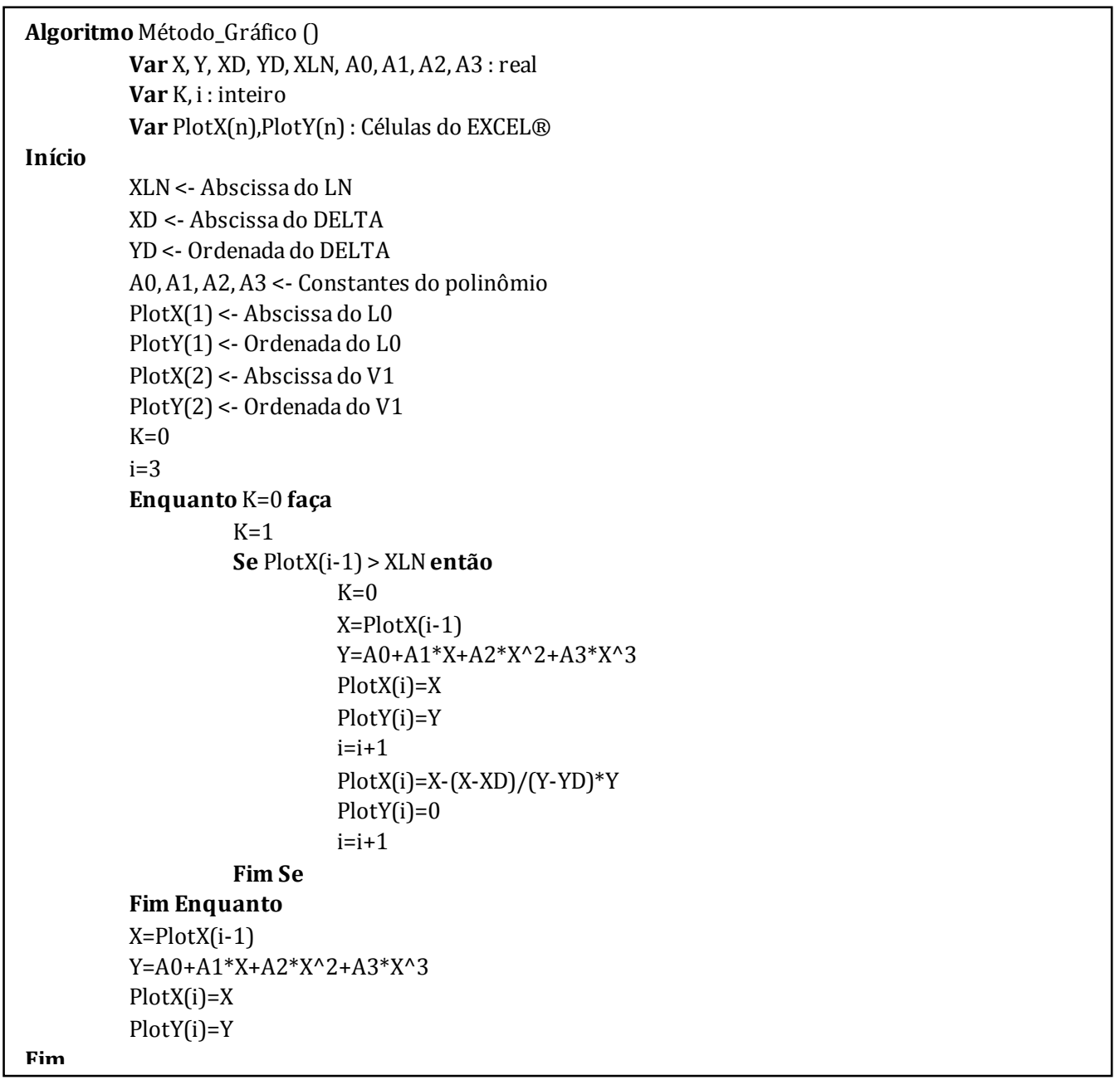

Figura 3 - Algoritmo utilizado no cálculo do número de estágios.

Finalmente, utiliza-se o EXCEL® para plotar a curva de retenção obtida pelo polinômio $\mathrm{Y}^{*}$ e os pontos (PlotX, PlotY) obtidos através do algoritmo apresentado, implementado no editor de Visual Basic.

\section{RESULTADOS DA IMPLEMENTAÇÃO NO EXCEL®}

O método algébrico de determinação do número de estágios é ilustrado em PERRY e CHILTON (1980) através de um exemplo de lixiviação em contracorrente de determinado minério. Neste exemplo, deseja-se lixiviar 420 toneladas por dia deste minério que contém 16,8\% de soluto, $16,5 \%$ de água e o restante de inertes. Dispõe-se de 200 toneladas por dia de água de lavagem com $1 \%$ de soluto em peso e a descarga da fase leve do processo deve conter $33 \%$ de solúveis. Além disso, deseja-se recuperar $88 \%$ de todo soluto alimentado. 
As figuras 4 e 5 mostram os resultados obtidos na resolução deste mesmo problema atra vés do EXCEL®, fazendo uso da metodologia e do algoritmo apresentados neste trabalho.

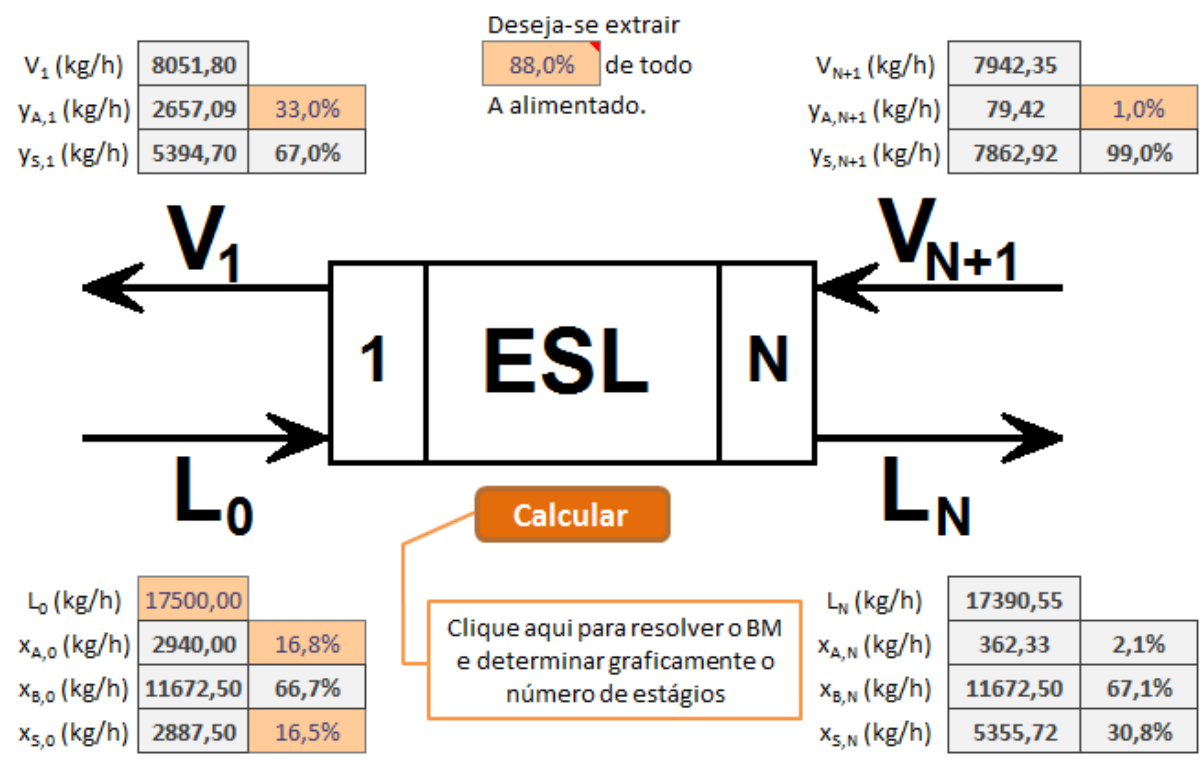

Figura 4 - Resultado da resolução do problema proposto através do programa.

As células em laranja representam os dados de entrada no programa e as células em cinza mostram os dados de saída, ou seja, os resultados dos cálculos realizados pelo programa, em $\mathrm{kg} / \mathrm{h}$. Estes resultados, e também os resultados obtidos por PERRY e CHILTON (1980), podem ser encontrados na tabela 1, em ton/dia.

Tabela 1 - Resultados obtidos por PERRY e CHILTON (1980) e por FERREIRA e MOTTA LIMA (2014) para o problema proposto

\begin{tabular}{|c|c|c|c|}
\hline Variável & $\begin{array}{c}\text { PERRY e CHILTON } \\
(1980)\end{array}$ & $\begin{array}{c}\text { FERREIRA e MOTTA } \\
\text { LIMA (2014) }\end{array}$ & D $(\%)$ \\
\hline$L_{N}($ ton/dia $)$ & 417,8 & 417,4 & 0,10 \\
\hline$\left(x_{A} \cdot L\right)_{N}($ ton/dia $)$ & 8,5 & 8,7 & 2,30 \\
\hline$\left(x_{S} \cdot L\right)_{N}($ ton/dia $)$ & 129,3 & 128,5 & 0,59 \\
\hline$V_{1}($ ton/dia $)$ & 194,0 & 193,2 & 0,39 \\
\hline$V_{N+1}($ ton/dia $)$ & 191,8 & 190,6 & 0,62 \\
\hline $\mathrm{N}$ & 4 & 4 & - \\
\hline
\end{tabular}




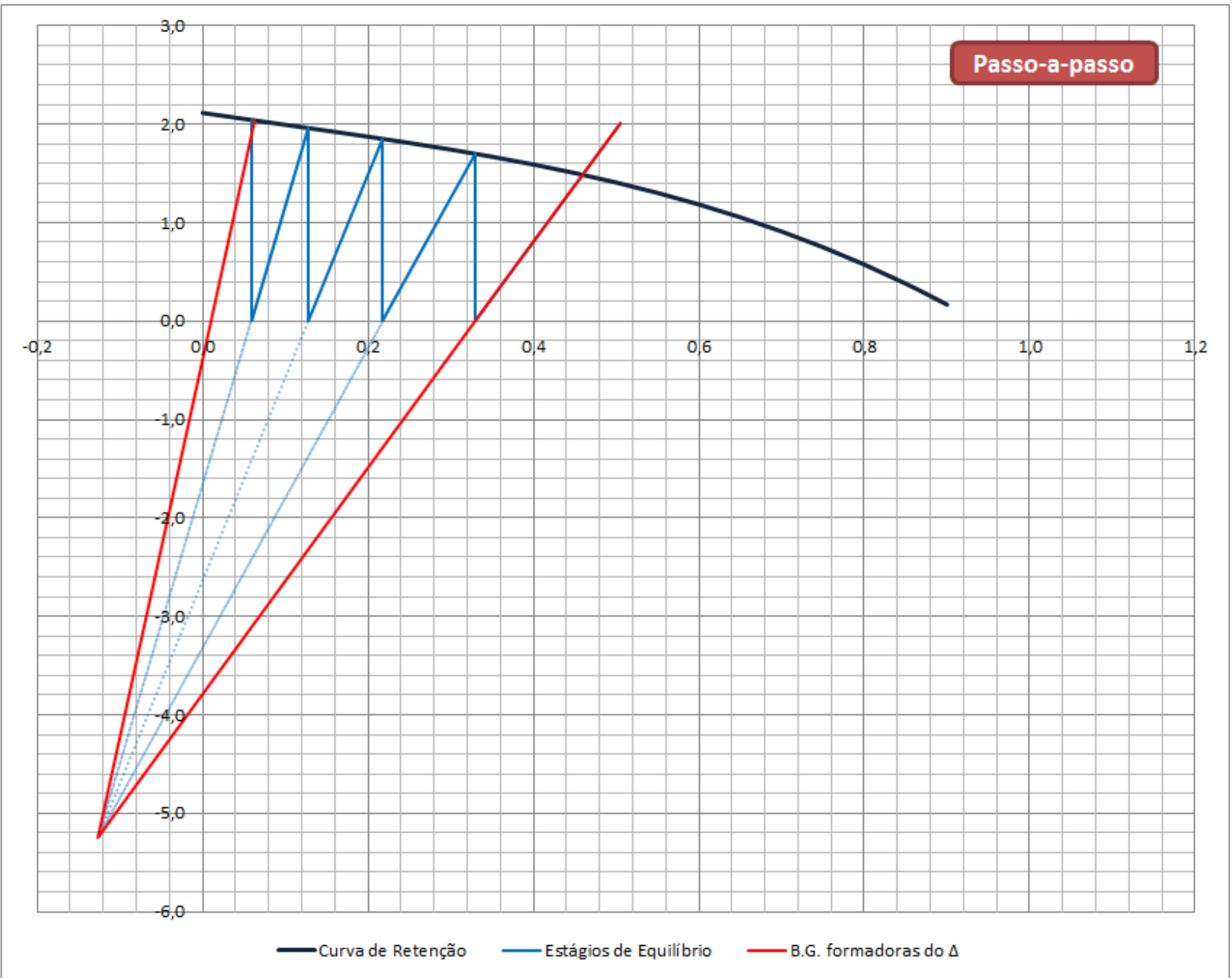

Figura 5 - Procedimento gráfico realizado através do programa.

Pode-se observar que os resultados obtidos através do uso da metodologia e do algoritmo apresentados aqui neste trabalho condizem com o esperado. $\mathrm{O}$ desvio em relação aos resultados obtidos por PERRY e CHILTON (1980) para o mesmo problema foram bastante pequenos e devemse aos desvios do modelo polinomial em relação aos dados experimentais. 


\section{CONCLUSÃO}

Pode-se observar que a ferramenta apresentada neste trabalho conduz efetivamente à solução de problemas de extração sólido-líquido em contracorrente. Além disso, a utilização do EXCEL® e seu SOLVER garante resultados confiáveis para os cálculos envolvidos.

Ainda, a aplicação desta ferramenta é de fácil manuseio mesmo para usuários que não tenham muita intimidade com este software, o que garante a eficácia em sua implementação. Esta vantagem se mostra importante, pois a programação realizada em Visual Basic e EXCEL® garante que mesmo os usuários com pouca experiência no uso de planilhas eletrônicas consigam interagir com o programa, podendo modificá-lo e operá-lo, sem muita dificuldade, conforme suas necessidades.

Finalmente, esta ferramenta mostrou-se capaz de contribuir de forma significativa no ensino de extração sólido-líquido.

\section{NOMENCLATURA}

$\mathrm{L}_{\mathrm{j}}$ - Corrente de sólidos $\left[\mathrm{MT}^{-1}\right]$;

$\mathrm{V}_{\mathrm{j}}$ - Corrente de solvente $\left[\mathrm{MT}^{-1}\right]$;

$\mathrm{x}_{\mathrm{i}, \mathrm{j}}$ - Fração do componente i na corrente j de sólidos;

$\mathrm{y}_{\mathrm{i}, \mathrm{j}}$ - Fração do componente i na corrente $\mathrm{j}$ de solvente;

A - Sólidos solúveis (soluto);

B - Sólidos inertes;

S - Solvente;

i - índice referente ao componente (A, B ou S);

j- índice referente ao estágio (1 a N).

\section{REFERÊNCIAS}

FOUST, A. S.; WENZEL, L. A.; CLUMP, C. W.; MAUS, L.; ANDERSEN, L. B. Princípios das Operações Unitárias. Rio de Janeiro: Guanabara Dois, 1982.

MOTTA LIMA, O. C.; PEREIRA, N. C. Apostila de Extração Sólido-Líquido. Maringá: Departamento de Engenharia Química - UEM, 2010.

PERRY, R. H.; CHILTON, C. H. Manual de Engenharia Química. Rio de Janeiro: Guanabara Dois, 1980. 